\title{
Vascular invasion in non-small cell lung carcinoma
}

\author{
T E Roberts, P S Hasleton, C Musgrove, R Swindell, R A M Lawson
}

\begin{abstract}
Aims: To determine if there is any correlation between vascular invasion and prognosis in non-small cell carcinoma of the lung; and to look specifically at invasion of vascular channels by tumour cells.

Methods: Eighty seven patients undergoing lobectomy or pneumonectomy for adenocarcinoma or squamous carcinoma were followed up for five years. The histological sections were studied for evidence of vascular invasion using an elastic van Gieson stain. The incidence of intimal fibrosis in arteries and veins was noted and the proportion with vascular invasion evaluated using a scoring system. The presence or absence of lymphatic permeation and tumour necrosis were noted. Survival data were analysed using the log rank test.
\end{abstract}

Results: The overall five year survival was $32 \%$. There were 64 squamous cell carcinomas and 23 adenocarcinomas. Vascular invasion was seen in $77 \%$ of patients and lymphatic invasion in $44 \%$. Neither the presence nor absence nor the proportion of blood vessels showing vascular invasion showed any relation to prognosis. Intimal fibrosis and tumour necrosis were unrelated to prognosis. Patients with lymphatic permeation had recurrence and died earlier than those without.

Conclusion: The presence of arterial or venous invasion by adenocarcinoma or squamous carcinoma of the lung was unrelated to survival; lymphatic permeation was associated with poor prognosis. The two common non-small cell lung cancers behaved differently from other solid tumours, where vascular invasion was a significant factor in determination of prognosis. The presence of intimal fibrosis was unrelated to prognosis.

Invasion of the lymphatic or vascular space is a feature of malignant neoplasms, allowing them to metastasise. This has been shown for Wilms's tumour, ${ }^{1}$ cervical carcinoma, ${ }^{2}{ }^{3}$ breast carcinoma, ${ }^{45}$ endometrial carcinoma, ${ }^{6}$ lung, ${ }^{78}$ oesophagus ${ }^{9}$ and adenocarcinoma of the colon and rectum, ${ }^{10} 11$ although by contrast, Jass et al found that invasion of rectal veins did not influence the survival in rectal carcinoma. ${ }^{12}$

This study was undertaken to see if there was a similar correlation between vascular invasion and prognosis in carcinoma of the lung and to look specifically at invasion of vascular channels by tumour cells, rather than combining both vascular and lymphatic channels under the same broad heading.

\section{Methods}

Eighty seven patients who had resections for lung cancer between 1979 and 1983, and for whom there was complete follow up data, were studied retrospectively. The operations were all carried out by a single surgeon (RAML) and in each there was no clinical or radiological evidence of metastasis at the time of surgery. The number of histological blocks taken from each tumour ranged between two and six (mean four). In most of the cases studied at least one tumour block had some adjacent lung tissue in the histological section.

Slides from each block were stained conventionally with haematoxylin and eosin and elastic van Gieson to permit clear visualisation of blood vessel laminae. ${ }^{13}$ Only patients with squamous cell and adenocarcinoma were studied as it is well recognised that small cell tumours invade the pulmonary vasculature early. ${ }^{14}$ Undifferentiated tumours were also not considered as it was felt that they comprise a very mixed group and should be studied separately. Lymphatic invasion was recorded as being present only if tumour cells were seen within areas which had a definite and clearly identifiable endothelial lining.

Vascular invasion was identified if tumour was seen within pulmonary arteries (figure) or veins. Blood vessels with invasion of the media but with an intact intima were not considered to have true vascular invasion. Two pathologists (PSH, CM) independently recorded tumour type and the nature of the vessels involved, together with the presence or absence of intimal fibrosis and tumour necrosis. They also evaluated, semiquantitatively, the proportion of vascular invasion using the following scale: $0=$ no vessels involved, $1=<33 \%$ vessels involved, $2=50 \%$ vessels involved, and $3=>66 \%$ vessels involved. Where there was disagreement between the two pathologists the case was reassessed jointly under a double headed microscope. Care was taken to exclude intrabronchial tumour spread. This was done by referring to the haematoxylin and eosin sections as well as insistence on an arterial or venous histological structure. Arterioles or venules which could be confused with lymphatics were not counted on this study.

In addition to the foregoing histological features for each patient, survival data were analysed using the log rank test. Statistical 


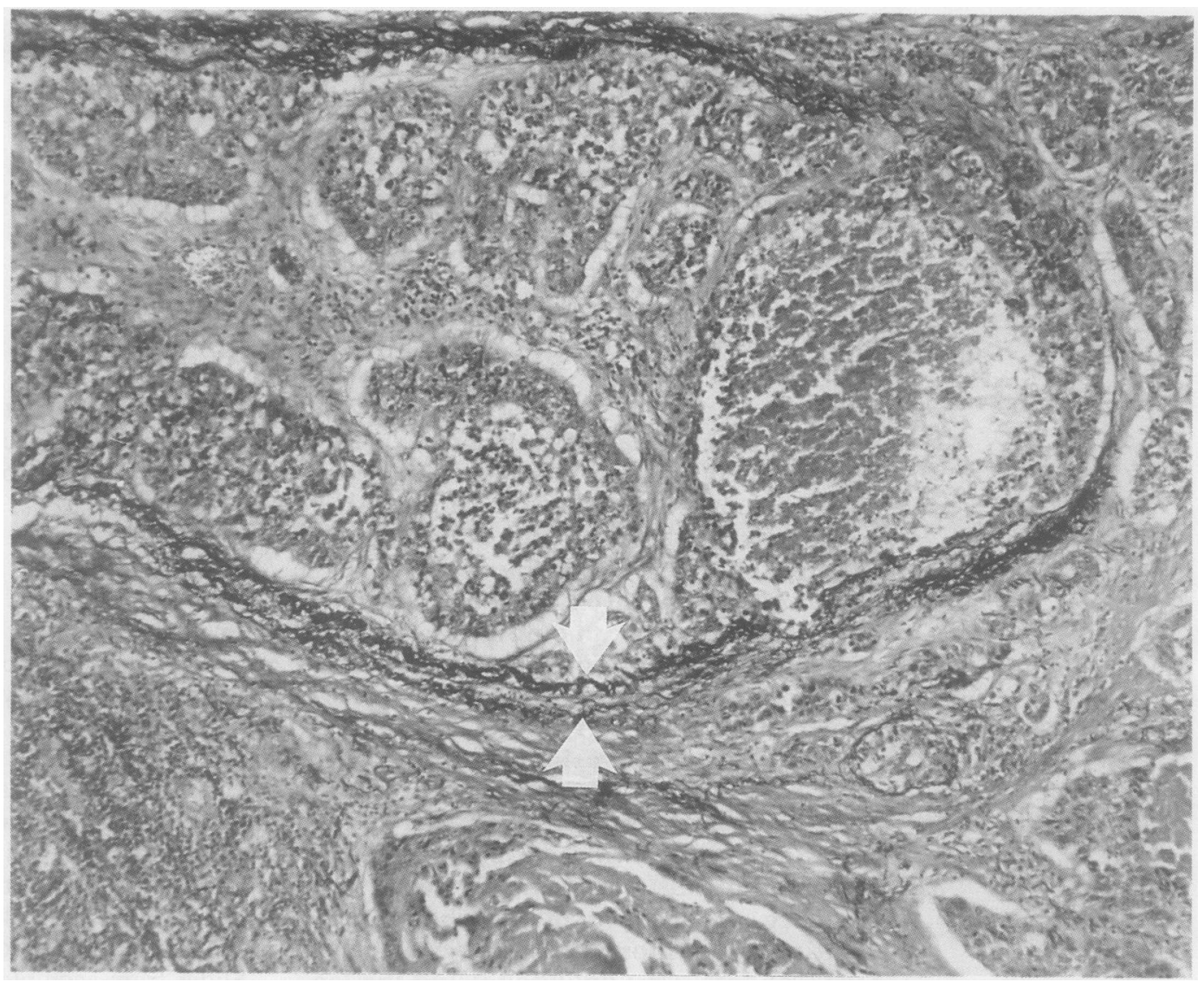

Figure Pulmonary artery (media well shown between arrows) infiltrated by squamous carcinoma (elastic van Gieson).

analysis of the contingency tables was performed using the $\chi^{2}$ test. The median duration of follow up was 92 months (range 14-141 months).

\section{Results}

The overall five year survival rate was $32 \%$. The number of tumours of each histological type and the incidence of vascular and lymphatic invasion is shown in table 1 . Vascular invasion was found in 67 of 87 patients (77\%) and lymphatic invasion was found in $\mathbf{3 8}$ of $\mathbf{8 7}$ patients $(44 \%)$. In patients with vascular invasion the pulmonary veins were more frequently affected than the arteries (data not shown).

There was no correlation between side nor site of tumour within the lung ( $p=0.5, p=$ 0.236 , respectively). There was a significant association between the presence of tumour within lymphatic channels and survival. Those patients with lymphatic invasion had recurrence and died earlier than those without $(p=$ 0.003 ). By contrast, neither the presence nor absence nor the proportion of blood vessels invaded by tumour was related to prognosis (table 2). The presence or absence of intimal fibrosis and tumour necrosis were also unrelated to prognosis (table 2).

Table 1 Tumour type

\begin{tabular}{lll}
\hline & \multicolumn{2}{c}{ Squamous } \\
\hline No of cases & 64 & 23 \\
No with vascular invasion & 49 & 18 \\
No with lymphatic invasion & 26 & 12 \\
\hline
\end{tabular}

\section{Discussion}

This study showed no correlation between presence of vascular invasion and survival in squamous and adenocarcinoma of the lung. However, lymphatic permeation was associated with a poor prognosis. The possibility that intimal fibrosis was responsible for retarding or preventing malignant infiltration and subsequent dissemination of cells was considered by examining the presence or absence of this change in each tumour and relating it to survival. There was no significant correlation. The difference between the observations in our study and those obtained by others with different tumour types may reflect the fact that most of the previous investigators have made no distinction between neoplastic invasion of lymphatics and blood vessels, and thus a strong correlation of survival with lymphatic invasion may have masked a lack of correlation with blood vessel disease. In addition, capillaries, being thin walled, can easily be confused with lymphatics, making their identification difficult in some cases. The five year survival rate of $32 \%$ in these patients studied is similar to the $30 \%$ reported in a similar cohort by the Edinburgh Lung Cancer Group. ${ }^{15}$

Table 2 Correlation between histological and physical variables and survival in carcinoma of the lung

\begin{tabular}{ll}
\hline Variable & Survival $p$ value \\
\hline Lymphatic invasion & 0.003 \\
Blood vessel invasion & 0.75 \\
Intimal fibrosis of vessels & 0.93 \\
Tumour necrosis & 0.76 \\
\hline
\end{tabular}


There is no way of demonstrating whether tumour cells identified within a vascular lumen are viable in vivo, and should a blood vessel be obliterated by tumour, the malignant cells may be relatively hypoxic and incapable of distant implantation and replication. At $77 \%$, the incidence of vascular invasion in this study of lung cancer is high, compared with $52 \%$ in rectal cancer, ${ }^{11} 17 \%$ of Wilms' tumour, ${ }^{1}$ and $25 \%$ of endometrial carcinoma. ${ }^{6}$ However, this figure is similar to that reported in bronchial tumours by Mosely and Dickson $(88 \%)^{7}$ and Kolin and Koutoulakis (58-87\%). ${ }^{8}$ This high incidence may reflect the relatively more vascular nature of the lung compared with other organs, the thin media of pulmonary blood vessels, or the lower intraluminal pressure of the pulmonary arterial bed, predisposing these vessels to invasion. As a practical point it is important that several tumour blocks are examined for presence of blood vessel disease. In a pilot study of 10 patients we found that the incidence of vascular invasion was grossly underestimated if only one tumour block was examined (unpublished data).

The mechanisms whereby tumours may metastasise are complex and not completely understood. The mechanical, enzymatic, and migratory characteristics of the cells seem to be important. ${ }^{14}$ Consequently, access to vascular channels is likely to be only one of many factors which may influence the outcome, and the Edinburgh Lung Cancer Group have shown that performance indices, such as the Karnosky score, are good prognostic indicators in both small cell and non-small cell tumours. ${ }^{15}$ The results of this study suggest that in terms of bloodborne metastases at least, carcinoma of the lung behaves differently from other common solid tumours.

1 Kumar APM, Hustu O, Fleming ID, et al. Capsular and Vascular invasion: important prognostic factors in Wilms' tumour. F Paed Surg 1975;10:301-8.

2 Barber HRK, Sommers SC, Rotterdam H, Kwon T. Vascular invasion as a prognostic factor in stage $1 \mathrm{~B}$ cancer of the cervix. Obstet Gynecol 1978;52:343-8.

3 van Nagel JR Jr, Donaldson ES, Wood EG, Parker JC Jr. The significance of vascular invasion and lymphocytic infiltration in invasive cervical cancer. Cancer 1978;41:228-34.

4 Bettleheim R, Munro Neville A. Lymphatic and vascular channel involvement within infiltrative breast carcinomas as a guide to prognosis at the time of primary surgical treatment. Lancet $1981 ; \mathrm{ii}: 631$.

5 Bettelheim R, Penman HG, Thornton-Jones H, Neville AM. Prognostic significance of peritumoral vascular invasion in breast cancer. Br $\mathcal{F}$ Cancer 1984;50:771-7.

6 Sivridis E, Buckley $\mathrm{CH}$, Fox H. The prognostic significance of lymphatic vascular space invasion in endometrial
of of lymphatic vascular space invasion in endometrial

adenocarcinoma. Br 7 Obstet Gynaecol 1987;94:991-4.
7 Mosely JM, Dickson DR. Vascular invasion in lung cancer. Am Rev Respir Dis 1960;82:807-9.

8 Kolin A, Koutoulakis T. Invasion of pulmonary arteries by bronchial carcinomas. Hum Pathol 1987;18:1165-71.

9 Edwards JM, Hillier VF, Lawson RAM, Moussalli H, Hasleton PS. Squamous carcinoma of the oesophagus: histological criteria and their prognostic significance. $\mathrm{Br} \mathcal{F}$ Cancer 1989;59:429-33.

10 Griffiths JD, McKinna HD, Rowbotham HD, Tsolakidis P, Salsbury AJ. Carcinoma of the colon and rectum: circulating malignant cells and five year survival. Cancer 1973;31:226-36.

11 Talbot IC, Ritchie S, Leighton MH, Hughes AO, Bussey HJR, Morson BC. The clinical significance of invasion of $\mathrm{HJR}$, Morson BC. The clinical significance of invas
veins by rectal cancer. Br $₹$ Surg 1980;67:439-42.

12 Jass JR, Love SB, Northover JMA. A new prognostic classification of rectal cancer. Lancet 1986;i:1303-6.

13 Hasleton PS, Heath D, Brewer DB. Hypertensive pulmonary vascular disease in states of chronic hypoxia. $\mathcal{f}$ Pathol Bacteriol 1968;95:431-40.

14 Greco FA, Oldham RK. Current concepts in cancer: small cell lung cancer. $N$ Engl $₹$ Med 1979;301:355-8.

15 Capewell S, Sudlow MF. Performance and prognosis in patients with lung cancer. Thorax 1990;45:951-6. 